\title{
Stimulus control in multiple variable-ratio schedules of reinforcement
}

\author{
LINDA HELLENTHAL and HENRY MARCUCELLA \\ Boston University, Boston, Massachusetts 02215
}

\begin{abstract}
One component of a multiple variable-ratio 150 variable-ratio 150 schedule remained unchanged while the reinforcement schedule of the other component was periodically changed to extinction and then returned to variable ratio 150 . When the reinforcement schedule of the changed component was an unmodified variable ratio 150 schedule, the magnitude of negative contrast during baseline recovery was equal to the magnitude of positive contrast observed during the previous change from multiple variable-ratio 150 variable-ratio 150 to multiple variable-ratio 150 extinction. When the schedule of the changed component was modified during baseline recovery so that responses terminating interresponse times greater than the average baseline interresponse time in the unchanged component were not reinforced, the magnitude of the unchanged-component response rate decrease was reduced. The magnitude of negative contrast was attenuated even though response and reinforcement rates in the variable component increased to levels at or above their prior multiple variable-ratio 150 variable-ratio 150 baseline levels. The results support a general theory that negative contrast results from both (1) the removal of certain positive-contrast-producing operations and (2) changes in the interresponse time-reinforcer relations that occur as a byproduct of schedule manipulations.
\end{abstract}

When the reinforcement schedule associated with one component (variable) of a multiple schedule of reinforcement is changed to extinction (EXT), the response-rate decrease in this component is accompanied by an increase in the response rate of the other (constant) component. When response rates have stab. ilized and the variable-component reinforcement schedule is subsequently reintroduced, the response-rate increase in this component is typically accompanied by a decrease in the constant-component response rate. These constant-component response rate changes are referred to as instances of positive and negative behavioral contrast (Reynolds, 1961a). The many possible interpretations of these effects (Bloomfield, 1969; Gamzu \& Schwartz, 1973; Reynolds, 1961b; Terrace, 1972) all view positive and negative contrast as causally related phenomena, i.e., the occurrence of negative contrast is attributed to the removal of the manipulation that was initially responsible for the occurrence of positive contrast.

Recently, Marcucella and MacDonall (1977) have presented considerable evidence in support of Schwartz's (1975) suggestion that the variables responsible for posi-

This paper is based on part of a dissertation by the first author, submitted to the Boston University Department of Psychology in partial fulfilment of the requirements for the $\mathrm{PhD}$ degree. The authors wish to thank Drs. G. Margolius and D. I. Mostofsky for their helpful comments. Reprints may be obtained from Dr. Linda Hellenthal, Bear River Community Mental Health Center, 198 N. Main Street, P.O. Box 683, Logan, Utah 84321. tive contrast are different from those which produce negative contrast. In a series of experiments, they first demonstrated that changing one component of mult VIVI schedule to extinction increased the frequency of long IRTs in that component, and second, that these long IRTs were selectively reinforced when the baseline schedule was reintroduced. Marcucella and MacDonall suggested that negative contrast results as an inductive effect of reinforcing these long IRTs. They then demonstrated that negative contrast could be eliminated by preventing the reinforcement of these long IRTs in the variable component during the transition from mult VI EXT to mult VI VI.

However, Marcucella and MacDonall's results may have depended more upon the formal properties of interval schedules than upon the changes in the obtained relative frequency of reinforcement for specific IRTs that occurred during the transition from mult VI EXT to mult VI VI. For example, in interval schedules, the theoretical probability that an IRT will be reinforced increases as the length of the IRT increases; i.e., the longer the pause between responses, the higher the probability that the response which terminates the pause will be reinforced. Thus, in interval schedules, long IRTs have a higher theoretical probability of being reinforced than do shorter IRTs.

The purpose of the present experiment was to assess the role of differing theoretical probabilities of reinforcement for specific IRTs by systematically replicating their second experiment using schedules in which IRTs of various lengths have an equal theore- 
tical probability of reinforcement. Specifically, the purpose of the present experiment was to demonstrate that the magnitude of negative contrast could also be significantly reduced by adding a pacing requirement to the variable component during the transition from mult VR 150 EXT to mult VR 150 VR 150. The pacing requirement specified that responses be reinforced only if they terminated IRTs equal to or less than the mean IRT of the constant component in the baseline mult VR 150 VR 150 schedule.

\section{METHOD}

Subjects

Three naive White Carneaux pigeons were maintained at $80 \%$ of their free feeding weights. Water was available only in the home cage.

\section{Apparatus}

The apparatus was a standard pigeon test chamber (Gerbrands Model G7311) containing one clear response key. The key was transilluminated by an Industrial Electronics Engineers in-line projector. Keypecks of at least $.15 \mathrm{~N}$ force were recorded; they operated a feedback relay located behind the front panel. The reinforcer consisted of $3.7 \mathrm{sec}$ access to mixed grain from a food hopper located below the key. During reinforcement periods, the key was dark and the food hopper was illuminated by two 28-V lamps. The houselight remained on until the end of the session except during reinforcement periods. A white-noise generator and an exhaust fan provided masking noise. Solid state programming and recording equipment was located in an adjacent room.

\section{Procedure}

Keypecks were reinforced in the presence of red and green key lights on a two-component multiple schedule with identical VR 150 schedules associated with each component. Keypecks were first continuously reinforced and then were reinforced on successively larger mean-valued variable-ratio schedules until the final VR 150 schedule was reached. The VR 150 ratio values were $449,48,176,4,112,96,4,209,401,48$, $241,16,48,281$, and 112 responses; they were multiplicative values of tape intervals which have been shown to maintain stable variable-interval schedule responding (Catania \& Reynolds, 1968). The components were $1 \mathrm{~min}$ long and alternated strictly for Bird 3760 and semirandomly for Bird 3774. For Bird 3802, the components alternated strictly during Conditions 1 through 7 and semirandomly during Conditions 8 through 12 . No blackout periods separated the components.

While the reinforcement schedule associated with the constant component (green) remained unchanged throughout the experiment, the schedule associated with the variable component (red) was periodically changed to EXT and then returned to either paced VR 150 or unpaced VR 150. Sessions were terminated after the delivery of 50 reinforcers during mult VR 150 VR $150+$ pacing and mult VR 150 VR 150 schedules. Sessions were terminated after the delivery of 25 reinforcers during mult VR $150 \mathrm{EXT}$. The variable-component schedule was changed when responding in both components appeared stable, and when no trends were evident in either direction. The order of conditions for each subject, the number of sessions at each condition, and the pacing values for each bird are shown in Table 1.

Pacing. Whenever the variable-component pacing requirement was in effect, a response was reinforced only if it terminated an IRT $\leqslant$ the mean constant-component IRT for the bird during the last five sessions of baseline mult VR 150 VR 150 (i.e., the reciprocal of the mean constant-component re-

Table 1

The Sequence of Conditions, the Number of Sessions at Each Condition, and the Mean Response, Reinforcement, and Relative Reinforcement Rate in Both Components for the Last Five Sessions of Each Condition

\begin{tabular}{|c|c|c|c|c|c|c|c|c|c|c|}
\hline \multirow[b]{2}{*}{ Subject } & \multirow{2}{*}{$\begin{array}{c}\text { Condi- } \\
\text { tion }\end{array}$} & \multicolumn{2}{|r|}{ Schedule } & \multirow[b]{2}{*}{ Session } & \multicolumn{2}{|c|}{ Responses/Min } & \multicolumn{2}{|c|}{$\mathbf{S}^{\mathbf{R}}$ Rate } & \multicolumn{2}{|c|}{ Relative $S^{R}$ Rate } \\
\hline & & C & $\mathbf{V}$ & & $\mathrm{C}$ & $\mathbf{V}$ & $\mathrm{C}$ & $\mathbf{V}$ & C & V \\
\hline 3760 & $\begin{array}{l}1 \\
2 \\
3 \\
4 \\
5 \\
6 \\
7\end{array}$ & $\begin{array}{l}\text { VR } 150 \\
\text { VR } 150 \\
\text { VR } 150 \\
\text { VR } 150 \\
\text { VR } 150 \\
\text { VR } 150 \\
\text { VR } 150\end{array}$ & $\begin{array}{l}\text { VR } 150 \\
\text { Ext } \\
\text { VR } 150 \\
\text { VR } 150 \\
\text { Ext } \\
\text { VR } 150 \text { paced } 0, .32 \mathrm{sec} \\
\text { VR } 150\end{array}$ & $\begin{array}{l}24 \\
42 \\
24 \\
23 \\
43 \\
41 \\
22\end{array}$ & $\begin{array}{l}300.1 \\
370.2 \\
292.5 \\
190.4 \\
372.8 \\
276.1 \\
252.2\end{array}$ & $\begin{array}{r}309.0 \\
93.3 \\
274.2 \\
224.7 \\
85.5 \\
287.9 \\
251.8\end{array}$ & $\begin{array}{l}1.92 \\
2.51 \\
1.91 \\
1.32 \\
2.43 \\
1.71 \\
1.53\end{array}$ & $\begin{array}{l}2.03 \\
1.77 \\
1.43 \\
1.89 \\
1.65\end{array}$ & $\begin{array}{r}.49 \\
1.00 \\
.52 \\
.48 \\
1.00 \\
.48 \\
.48\end{array}$ & $\begin{array}{l}.48 \\
.52 \\
.52 \\
.52\end{array}$ \\
\hline 3802 & $\begin{array}{r}1 \\
2 \\
3 \\
4 \\
5 \\
6 \\
7 \\
8 \\
9 \\
10 \\
11 \\
12\end{array}$ & $\begin{array}{l}\text { VR } 150 \\
\text { VR } 150 \\
\text { VR } 150 \\
\text { VR } 150 \\
\text { VR } 150 \\
\text { VR } 150 \\
\text { VR } 150 \\
\text { VR } 150 \\
\text { VR } 150 \\
\text { VR } 150 \\
\text { VR } 150 \\
\text { VR } 150\end{array}$ & $\begin{array}{l}\text { VR } 150 \\
\text { Ext } \\
\text { VR } 150 \\
\text { VR } 150 \\
\text { Ext } \\
\text { VR } 150 \text { paced } 0, .25 \mathrm{sec} \\
\text { VR } 150 \\
\text { VR } 150 \\
\text { Ext } \\
\text { VR } 150 \\
\text { Ext } \\
\text { VR } 150 \text { paced } 0, .31 \mathrm{sec}\end{array}$ & $\begin{array}{r}25 \\
33 \\
24 \\
5 \\
38 \\
27 \\
8 \\
18 \\
61 \\
20 \\
35 \\
20\end{array}$ & $\begin{array}{l}163.1 \\
356.2 \\
188.8 \\
230.8 \\
293.5 \\
268.6 \\
265.6 \\
255.5 \\
263.8 \\
199.5 \\
193.0 \\
183.1\end{array}$ & $\begin{array}{r}168.8 \\
18.0 \\
176.3 \\
228.6 \\
31.1 \\
225.9 \\
245.0 \\
249.8 \\
10.2 \\
184.1 \\
9.2 \\
165.4\end{array}$ & $\begin{array}{l}1.16 \\
2.38 \\
1.26 \\
1.50 \\
1.98 \\
1.79 \\
1.70 \\
1.68 \\
1.78 \\
1.36 \\
1.29 \\
1.26\end{array}$ & $\begin{array}{l}1.15 \\
1.16 \\
1.47 \\
1.49 \\
1.57 \\
1.64 \\
1.22 \\
1.11\end{array}$ & $\begin{array}{r}.50 \\
1.00 \\
.52 \\
.51 \\
1.00 \\
.55 \\
.52 \\
.51 \\
1.00 \\
.53 \\
1.00 \\
.53\end{array}$ & $\begin{array}{l}.50 \\
.48 \\
.49 \\
.45 \\
.48 \\
.49 \\
.47 \\
.47\end{array}$ \\
\hline 3774 & $\begin{array}{l}1 \\
2 \\
3 \\
4 \\
5 \\
6\end{array}$ & $\begin{array}{l}\text { VR } 150 \\
\text { VR } 150 \\
\text { VR } 150 \\
\text { VR } 150 \\
\text { VR } 150 \\
\text { VR } 150\end{array}$ & $\begin{array}{l}\text { VR } 150 \\
\text { Ext } \\
\text { VR } 150 \text { paced } 0, .24 \mathrm{sec} \\
\text { VR } 150 \\
\text { Ext } \\
\text { VR } 150\end{array}$ & $\begin{array}{l}22 \\
92 \\
25 \\
17 \\
27 \\
25\end{array}$ & $\begin{array}{l}248.9 \\
373.4 \\
306.0 \\
299.4 \\
366.9 \\
315.6\end{array}$ & $\begin{array}{r}256.5 \\
39.4 \\
293.6 \\
294.7 \\
29.1 \\
290.1\end{array}$ & $\begin{array}{l}1.77 \\
2.46 \\
2.09 \\
2.04 \\
2.48 \\
2.17\end{array}$ & $\begin{array}{l}1.97 \\
1.98 \\
\\
2.02\end{array}$ & $\begin{array}{r}.51 \\
1.00 \\
.51 \\
.51 \\
1.00 \\
.52\end{array}$ & $\begin{array}{l}.49 \\
.49 \\
.48\end{array}$ \\
\hline
\end{tabular}

Note-In Conditions 3 and 6 the mean data are presented for Sessions 20-24. $C=$ constant; $V=$ variable. 
sponse rate in seconds). Thus, whenever a variable-component reinforcer was scheduled, the variable-component pacing contingency required that the time interval separating the final two responses of the ratio be $<$ the specified value. Note that the pacing contingency was in effect only for variablecomponent responses and was removed when the variablecomponent response rate reached the level of its previous baseline and little or no pausing was evident. A schedule change from mult VR 150 EXT to mult VR 150 VR 150 with the pacing requirement will be referred to as the paced return to baseline, and a change from mult VR 150 EXT to mult VR 150 VR 150 without a pacing requirement will be referred to as an unpaced return to baseline.

\section{Control}

The mean IRT pacing value, based on baseline constantcomponent performance, was chosen in order to eliminate the reinforcement of long variable-component IRTs without producing high response rates in the constant component. Since the distribution of IRTs generated on VR schedules is positively skewed, a pacing value equal to the mean IRT should prevent long IRTs in the variable component from being reinforced without disrupting the reinforced IRT distribution simultaneously in effect in the constant component.

A control experiment was conducted in order to examine whether adding the pacing contingency to one component of a mult VR 150 VR 150 schedule would increase the constantcomponent response rates above baseline levels. If the introduction of the pacing contingency in the variable component reinforced short IRTs, and thus produced a high response rate, this high response rate in turn could have occurred in the constant component by induction (Hemmes \& Eckerman, 1972).

Four birds, two experimentally naive White Carneaux pigeons (5500 and 3123) and two of the pigeons used in the main experiment ( 3760 and 3802$)$, were used in the control experiment.

For the two naive birds, keypecks were first continuously reinforced, and then were reinforced on successively larger mean-valued variable-ratio schedules until the final VR 150 schedule was reached. Keypecks for all four birds were then reinforced in the presence of red and green key lights on a twocomponent multiple schedule with identical VR 150 schedules associated with each component. The VR 150 tape values were the same as those used in the main experiment. The components were $1 \mathrm{~min}$ long and alternated strictly for Birds 3760,5500 , and 3123 , and semirandomly for Bird 3802 . No blackout periods separated the components.

While the reinforcement schedule associated with the constant component (green) remained unchanged throughout the experiment, the schedule associated with the variable component (red) was changed to VR $150+$ pacing for 20 sessions and then returned to VR 150. The variable-component schedule was changed to VR 150 + pacing directly following the mult VR 150 VR 150 baseline condition. All sessions were terminated after the delivery of 50 reinforcers. The pacing values for each bird were: Bird 5500, $0.28 \mathrm{sec}$; Bird 3123, $0.39 \mathrm{sec}$; Bird $3760,0.23 \mathrm{sec}$; Bird 3802, $0.33 \mathrm{sec}$.

\section{RESULTS}

Figure 1 shows the response rates in both the constant (closed circles) and the variable (open circles) components for each bird during the last 5 sessions of baseline mult VR 150 VR 150 and during the first 10 sessions and the last 10 sessions of mult VR 150 EXT. The mean response rate in the constant component for each bird during the last 5 sessions of baseline mult VR 150 VR 150 is represented in Figure 1 as a dashed horizontal line. The conditions shown in the left panel preceded the unpaced returns to mult VR 150 VR 150 , and the conditions on the right preceded the transitions to mult VR 150 VR $150+$ pacing.

Figure 1 shows that, during the transition from mult VR 150 VR 150 to mult VR 150 EXT, positive contrast was obtained for all birds. During Conditions 9 and 11 for Bird 3802, however, the magnitude of positive contrast was small and dissipated with extended exposure to mult VR 150 EXT.

Figure 2 shows the normalized response rates in both the constant (closed circles) and the variable (open circles) components per session during the transition from mult VR 150 EXT to mult VR 150 VR 150. The conditions on the left represent the unpaced returns to baseline, and the conditions on the right represent the paced returns to baseline. The normalized response rates in the constant and variable component were calculated by dividing the response rate in each component for each session by the mean response rate in the constant component during the last five sessions of the preceding mult VR $150 \mathrm{EXT}$ condition. To allow a comparison with the baseline mult VR 150 VR 150 condition preceding each mult VR 150 EXT condition, the mean normalized response rates in the constant component during the last five sessions of baseline mult VR 150 VR 150 are represented in Figure 2 as dashed horizontal lines. These rates were also normalized by dividing by the mean response rate in the constant component during the last five sessions of mult VR 150 EXT. Thus, all response rates are expressed as percentages of the mean response rate in the constant component during the last five sessions of mult VR 150 EXT. The vertical arrow in each panel marks the point at which the pacing contingency in the variable component was removed.

Figure 2 shows that the response rates in the constant component decreased somewhat regardless of whether (1) the pacing contingency was in effect or (2) in agreement with Reynolds and Limpo (1968), positive contrast was present prior to the change from mult VR 150 EXT to mult VR 150 VR 150. However, for each pigeon, the percentage decrease in the response rate in the constant component during the paced return to mult VR 150 VR 150 was smaller than that observed during the unpaced return to mult VR 150 VR 150. In those situations where positive contrast was still present during mult VR 150 EXT, the introduction of the pacing contingency prevented the response rates in the constant component from declining to the level of the previous mean baseline response rate in the constant component. Instead, the response rates in the constant component for all birds remained above baseline levels for a minimum of 25 sessions and were unaffected by the removal of the pacing contingency (arrows). 


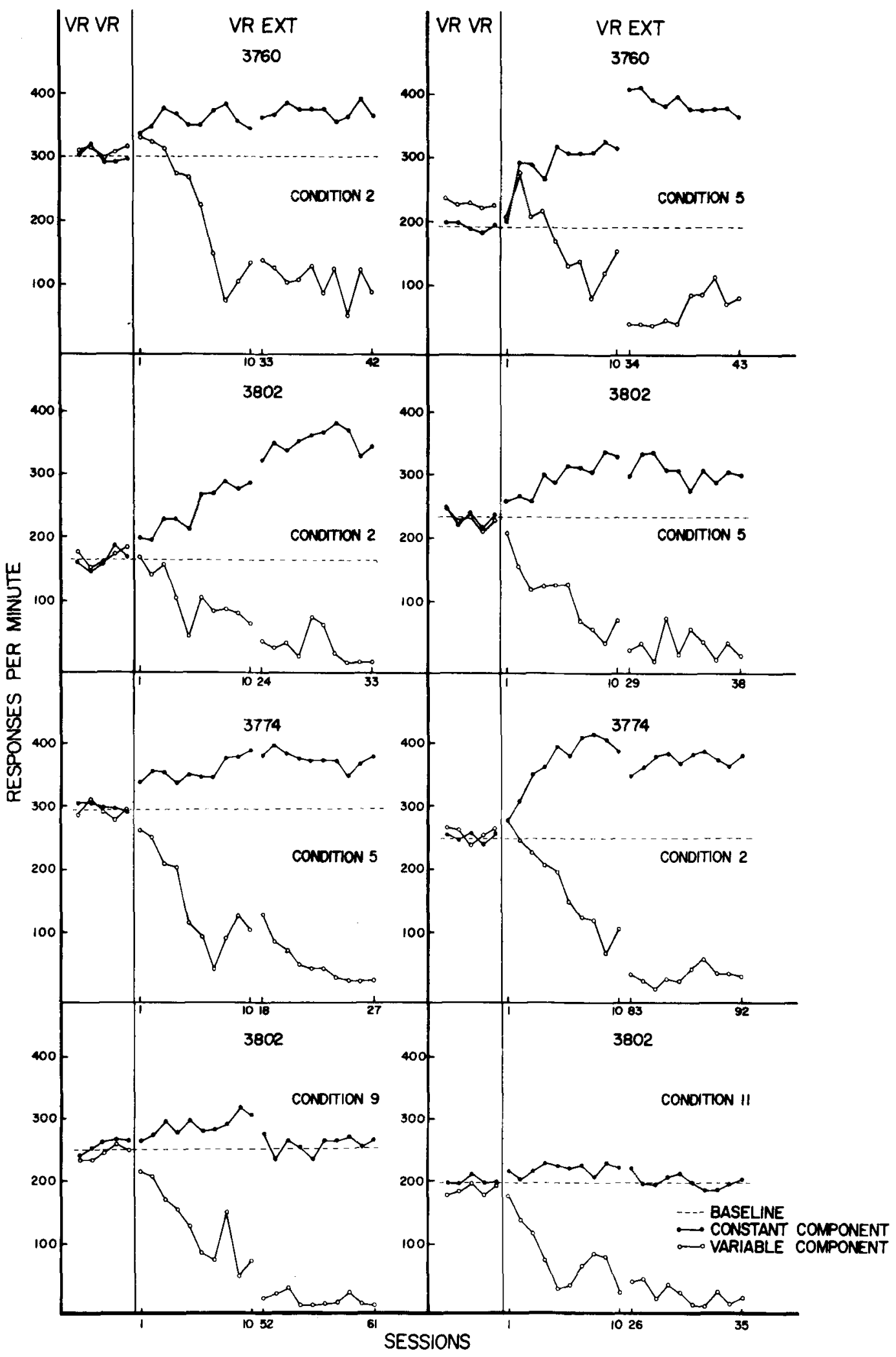

Figure 1. Response rates per minute for each bird in both the constant (closed circles) and the variable component (open circles) during the last 5 sessions of baseline mult VR 150 VR 150 (left panels) and the first 10 sessions and the last 10 sessions of mult VR 150 EXT (right panels). The dashed horizontal line represents the mean constant-component response rate during the last 5 sessions of baseline mult VR 150 VR 150. Conditions 2 (Birds 3760 and 3802), 5 (Bird 3774), and 9 (Bird 3802 ) preceded the unpaced returns to mult VR 150 VR 150. Conditions 5 (Birds 3760 and 3802), 2 (Bird 3774), and 11 (Bird 3802 ) preceded the transitions to mult VR 150 VR $150+$ pacing. 


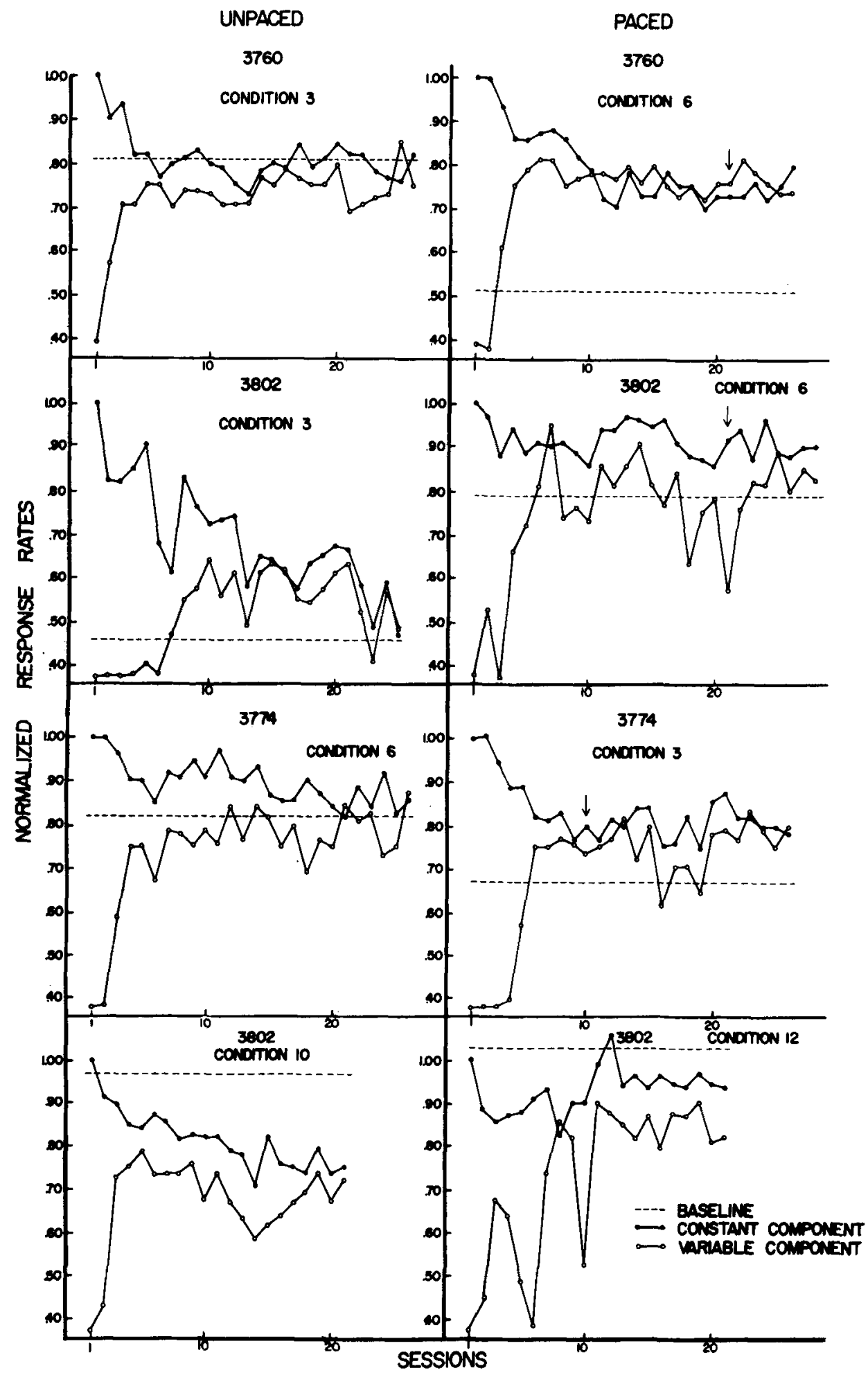

Figure 2. Normalized response tates in both the constant (closed circles) and the variable component (open circles) by sessions during the first 25 sessions of the paced and unpaced retums from mult VR 150 EXT to mult VR 150 VR 150 . Conditions 3 (Birds 3760 and 3802), 6 (Bird 3774), and 10 (Bird 3802) represent the unpaced retums to baseline, and Conditions 6 (Birds 3760 and 3802), 3 (Bird 3774), and 12 (Bird 3802) represent the paced returns to baseline. The normalized constant and variable-coimponent response rates were calculated by dividing the response rate in each component for each session by the mean constent-component response rate during the last 5 sessions of the preceding $\mathrm{mult}$ VR 150 EXT condition. The mean nomalized constant-component response rates during the last 5 sessions of baseline mult VR 150 VR 150 are represented by dashed horizontal lines. These rates were also normalized by dividing by the mean constant-component response rate during the last 5 sessions of mult VR 150 EXT. 
Figure 2 shows (Bird 3802, Conditions 10 and 12) that the decrease in the response rates in the constant component during the paced return to baseline was less than that obtained during the unpaced return to mult VR 150 VR 150, even when positive contrast had dissipated during mult VR 150 EXT.

The results cannot be attributed to the order in which the pigeons were exposed to the paced and unpaced conditions, since Birds 3760 and 3802 were first exposed to the unpaced condition and Bird 3774 to the paced condition.

Table 1 shows the order of conditions for each subject, the number of sessions at each condition, the mean response rates, reinforcement rates (reinforcers/ $\mathrm{min}$ ), and relative reinforcement rates in the constant and variable components for the last five sessions of each condition. Table 1 also shows, for each bird, the minimum IRT required for reinforcement when the pacing contingency in the variable component was in effect. For instance, for Bird 3760, Condition 6, the pacing requirement of $0,0.32 \mathrm{sec}$ indicates that variable component responses terminating IRTs $\leqslant$ $.32 \mathrm{sec}$ (to the limit of 0 ) were reinforced.

The data of Table 1 demonstrate that during the paced returns to baseline when positive contrast was still occurring, the response rates in the constant com. ponent remained above their previous mult VR 150 VR 150 baseline levels despite an increase in the response and reinforcement rates in the variable component to (Bird 3802) or above (Birds 3760 and 3774) baseline levels. While the absolute reinforcement rates in the variable components for two birds (3760 and 3774) increased above baseline levels during the paced returns to baseline, the data of Table 1 show that the relative reinforcement frequencies in the variable component for all three birds during the paced returns from mult VR 150 EXT to baseline were approximately the same as those previously observed during baseline mult VR 150 VR 150. Thus, despite the fact that, following a paced return from mult VR 150 EXT to mult VR 150 VR 150, the relative reinforcement frequencies in the variable component for all three birds eventually re. turned to prior baseline levels, the response rates in the constant component remained above baseline levels. The data of Table 1 show that a similar increase in the relative reinforcement frequencies in the variable component during the unpaced returns to baseline was accompanied by a decrease in the response rates in the constant component to (Bird 3760) or very near (Birds 3802 and 3774) baseline levels.

\section{Control}

Adding the pacing requirement to the variable component after stable responding had occurred during mult VR 150 VR 150 did not increase the response rates in either the constant or the variable component. Response rates in the constant component during mult VR
150 VR 150 + pacing remained approximately at or below their baseline mult VR 150 VR 150 levels. For three birds, response rates in the variable component during mult VR 150 VR 150 + pacing also remained at (Bird 3123) or slightly below (Birds 5500 and 3802) baseline levels. Although the response rates in the variable component increased temporarily above baseline levels for Bird 3760, the average response rate in the variable component during mult VR 150 VR 150 + pacing remained at the baseline level.

Removing the pacing contingency did not increase response rates in the constant component. The response rates in the constant component for all four birds remained at baseline levels, while the response rates in the variable component remained at (Birds 5500 and 3760 ) or slightly below (Bird 3123) baseline levels for three birds. The response rates in the variable component for Bird 3802 increased slightly when the pacing contingency was removed, but response rates in this component had returned to baseline levels by Session 6 .

\section{DISCUSSION}

The results of the present experiment demonstrated that the magnitude of negative contrast that was observed during the transition from mult VR 150 EXT to mult VR 150 VR 150 could be significantly reduced by selectively reinforcing variable-component IRTs $\leqslant$ the mean baseline constant-component IRT.

The results cannot be attributed to a difference in baseline response rates which preceded the paced vs. the unpaced returns from mult VR 150 EXT to mult VR 150 VR 150. The baseline response rates prior to the paced return from mult VR 150 EXT to mult VR 150 VR 150 were either lower (Bird 3760) or higher (Bird 3802, Condition 6) than the baseline response rates preceding the unpaced return to baseline. Yet, for both birds, the constant component response rates remained above prior mult VR 150 VR 150 baseline levels during the paced reintroduction of mult VR 150 VR 150, whereas during the unpaced return to baseline the constant component response rates fell to (Bird 3760) or very near (Bird 3802 , Condition 3) baseline levels.

The results cannot be attributed to a decrease in constant component response rates due to extended exposure to mult VR 150 EXT. Approximately the same number of discrimination-training sessions preceded the paced and unpaced returns to baseline for Birds 3760 and 3802 . Yet, for these birds, the percentage magnitude of negative contrast was reduced during the paced returns to baseline. For Bird 3774 , the number of discrimination-training sessions preceding the paced return to baseline was greater than that preceding the unpaced return to baseline. Yet the percentage magnitude of negative contrast for 
this bird was greater during the unpaced than during the paced return to baseline.

Thus the present results provide additional support for the hypothesis that changes in the obtained relative frequency of reinforcement for specific IRTs, occurring during the transitions between steady states of responding, determine, at least in part, the magnitude of negative contrast (Marcucella \& MacDonall, 1977). First, the present study demonstrated that the addition of the pacing procedure to ratio schedules in which IRTs of various lengths have equal theoretical probabilities of reinforcement also attenuated negative contrast. Second, the results of the present study suggest that Marcucella and MacDonall's account of negative contrast is applicable to schedules which generate high rates of behavior. For example, at first glance, it might appear that the hypothesis that negative contrast is due to the selective reinforcement of long IRTs may not be applicable to ratio schedules, because these schedules tend to differentially reinforce short IRTs even though, theoretically, IRTs of various lengths have an equal probability of reinforcement. Because responses reinforced on ratio schedules tend to occur in bursts, it is likely that the response requirement will be met during a burst, and as a result more short than long IRTs are actually reinforced (Morse, 1966).

If ratio schedules differentially reinforce short IRTs, how can an account of negative contrast in terms of the selective reinforcement of long IRTs explain the occurrence of negative contrast in ratio schedules? The answer is that any reinforcement schedule can differentially reinforce only those IRTs which are actually emitted by the organism. If responses are reinforced on a ratio schedule and only long IRTs are emitted, then only long IRTs will be reinforced. The present authors argue that this is exactly what happens during the transition from mult VR EXT to mult VR VR. When the VR schedule is first reintroduced, the pigeons emit mainly long IRTs in the variable component because of the previous history of extinction in that component. Thus, these long IRTs will be reinforced and increase in frequency. Negative contrast results as an inductive effect of reinforcing these long IRTs. Bursts of responding may again occur and be differentially reinforced, but only after (1) responding in the variable component has recov. ered somewhat and (2) the disruption of the relationship between the reinforced and unreinforced IRT distributions of the constant component.

Direct support for this interpretation is lacking because, unlike Marcucella and MacDonall (1977), the present authors did not record changes in the IRT distributions. However, indirect support was provided by showing that the magnitude of negative contrast was reduced when the reinforcement of long IRTs in the variable component was prevented during the transition from mult VR EXT to mult VR VR.

In summary, the present results support the hypothesis that the difference in magnitude of negative contrast relative to the magnitude of positive contrast obtained during the paced vs. unpaced returns to baseline was due to the elimination of the direct reinforcement of responses terminating long IRTs in the variable component (Marcucella \& MacDonall, 1977).

\section{REFERENCES}

Bloomfield, T. M. Behavioral contrast and the peak shift. In R. M. Gilbert \& N. S. Sutherland (Eds.), Animal discrimination learning. New York: Academic Press, 1969. Pp. 215-241.

Catania, A. C., \& Reynolds, G. S. A quantitative analysis of the responding maintained by interval schedules of reinforcement. Journal of the Experimental Analysis of Behavior, $1968,11,327-383$.

Gamzu, E., \& Schwartz, B. The maintenance of key pecking by stimulus contingent and response independent food presentation. Journal of the Experimental Analysis of Behavior. 1973, 19, 65-72.

Hemmes, N. S., \& Eckerman, D. A. Positive interaction (induction) in multiple variable interval, differential reinforcement of high rate schedules. Journal of the Experimental Analysis of Behavior, 1972, 17, 51-57.

Marcucella, H., \& MacDonall, J. S. A molecular analysis of multiple schedule interactions: Negative contrast. Journal of the Experimental Analysis of Behavior, 1977, 28, 71-82.

MoRsE, W. H. Intermittent reinforcement. In W. K. Honig (Ed.), Operant behavior: Areas of research and application. New York: Prentice-Hall, 1966. Pp. 52-108.

Reynozds, G. S. An analysis of interactions in a multiple schedule. Journal of the Experimental Analysis of Behavior, $1961,4,107-117$. (a)

ReYnolds, G. S. Behavioral contrast. Journal of the Experimental Analysis of Behavior, 1961, 4, 57-71 (b)

Reynolds, G. S., \& Limpo, A. J. Negative contrast after prolonged discrimination maintenance. Psychonomic Science, $1968,10,323-324$.

SchwarTz, B. Discriminative stimulus location as a determinant of positive and negative behavioral contrast in the pigeon. Journal of the Experimental Analysis of Behavior, 1975, 23, 167.176.

TERRACE, H. S. Byproducts of discrimination learning. In G. Bower \& J. Spence (Eds.), The psychology of learning and motivation. New York: Academic Press, 1972. Pp. 195-265.

(Received for publication April 12, 1977; revision accepted November 5, 1977.) 